\title{
A Proteomic Approach to Screening of Dynamic Changes in Detergent- Resistant Membranes from Activated Human Primary T Cells
}

\author{
Kristine Moltu ${ }^{1,2}$, Elisa Bjørgo ${ }^{1,2}$, Therese Solstad ${ }^{2 \#}$, Torunn Berge ${ }^{1,2}$, Bernd Thiede ${ }^{2}$ and Kjetil Taskén ${ }^{1,2,3 *}$ \\ ${ }^{1}$ Centre for Molecular Medicine Norway, Nordic EMBL Partnership, University of Oslo and Oslo University Hospital, Norway \\ ${ }^{2}$ Biotechnology Centre, University of Oslo, Norway \\ ${ }^{3}$ Department of Infectious Diseases, Oslo University Hospital, Oslo, Norway \\ \#Present address: The Norwegian Medicines Agency, Oslo, Norway
}

\begin{abstract}
Upon stimulation of the T cell receptor, proteins involved in proximal T cell signaling gather in lipid rafts to guide external stimuli to the intracellular compartment through the formation of signaling complexes. The rearrangement of lipid rafts upon engagement of the T cell receptor is crucial to ensure a rapid and efficient signal transduction. To assess lipid raft protein composition, we here provide a qualitative mass spectrometric characterization of the detergentresistant membrane (DRM) proteome in human primary T cells, identifying a total of 425 proteins. Furthermore, we have addressed proteins solely associated with DRMs in resting T cells and proteins solely observed in DRMs upon engagement of the T cell receptor. Only a small group of proteins were found to be exclusively located to DRMs under either of these conditions, indicating that the protein composition of DRMs in stimulated cells versus resting cells is remarkably stable. Classification of the 425 proteins identified in DRMs into functional categories revealed a particularly high occurrence of cytoskeletal proteins, indicating that DRMs remain attached to the underlying cortical actin cytoskeleton upon activation of T cells.
\end{abstract}

Keywords: Detergent-resistant membranes; Lipid rafts; Proteomics; T lymphocytes

Abbreviations: \#: Number; 2D: Two-Dimensional; APC: Antigen Presenting Cell; ATP: Adenosine Triphosphate; cAMP: Cyclic Adenosine Monophosphate; Cdc42: Cell Division Cycle 42; CIN85: Cbl-Interacting Protein of $85 \mathrm{kDa}$; Csk: C-terminal Src Kinase; DRM: Detergent-Resistant Membrane; ER: Endoplasmic Reticulum; GO: Gene Ontology; GPI: Glycosylphosphatidyl Inositol; HLA: Human Leukocyte Antigen; HRP: Horseradish Peroxidase; ICAM: Intercellular Adhesion Molecule; ILF3: Interleukin Enhancer-Binding Factor 3; ITAM: Immunoreceptor Tyrosine-Based Activation Motif; LAT: Linker of Activated T Cells; LFA: Lymphocyte Function-Associated Antigen; LTQ: Linear Quadrupole Ion Trap; MHC: Major Histocompatibility Complex; O-GlcNAc: O-linked N-Acetylglucosamine; OGP: n-OctylBeta-D-Gluco-Pyranoside; PAG: Phosphoprotein Associated with Glycosphingolipid-Enriched Microdomain; PAGE: Polyacrylamide Gel Electrophoresis; PKA: Protein Kinase A; PTM: Post-Translational Modification; PVDF: Polyvinylidene Fluoride; SFK: Src Family Kinase; STRAP: Software Tool for Researching Annotations of Proteins; STRING: Search Tool for the Retrieval of Interacting Genes/Proteins; TCR: T Cell Receptor; Th. $\mathrm{M}_{\mathrm{w}}$ : Theoretical Molecular Mass; UniProtKB acc.: UniProt Knowledgebase Accession Entry; VDAC: VoltageDependent Anion Selective Channel; ZAP-70: Zeta-Chain-Associated Protein of $70 \mathrm{kDa}$

\section{Introduction}

Lipid rafts are specialized plasma membrane domains enriched in cholesterol, sphingolipids and glycosylphosphatidyl inositol (GPI)-anchored proteins [1]. They are thought to function as signaling platforms playing an important role in signal transduction by gathering a variety of signaling proteins and thus facilitating the coordinated formation of numerous signaling complexes. Lipid rafts are also considered to be involved in cellular processes such as targeted protein trafficking, toxin- and virus entry, apoptosis and immune responses [2,3]. In particular, proteins involved in $\mathrm{T}$ cell signaling have been found to be constitutively present in lipid rafts, including key adaptor proteins such as LAT (linker for activation of T cells) and
PAG (phosphoprotein associated with glycosphingolipid-enriched microdomain). Furthermore, tyrosine kinases and other signaling molecules are recruited to lipid rafts upon $\mathrm{T}$ cell activation [3] and the integrity of these microdomains has also been proven essential for $\mathrm{T}$ cell activation to occur [4].

Activation of $\mathrm{T}$ cells is dependent on multiple interactions between antigen presenting cells (APCs) and molecules on the surface of the $\mathrm{T}$ cells. The $\mathrm{T}$ cell immune response is sustainable only if a second signal occurs in addition to primary activation of the $\mathrm{T}$ cell receptor (TCR). The second signal is typically elicited by ligands B7-1 or B7-2 on APCs through interaction with the co-receptor CD28, resulting in optimal interleukin-2 (IL-2) secretion, extensive clonal expansion and differentiation [5]. Engagement of the TCR and CD28 receptors activates members of the Src family kinases (SFKs), in particular Lck and FynT, which subsequently phosphorylate the immunoreceptor tyrosine-based activation motifs (ITAMs) present in the TCR/CD3 signaling complex. This, in turn, recruits the Syk family kinase ZAP-70 (zeta-chain-associated protein of $70 \mathrm{kDa}$ ), which becomes fully active upon Lck-mediated phosphorylation. ZAP-70 plays an essential role with respect to further propagation of the signal downstream of the TCR by phosphorylating the adapter protein LAT to aid recruitment of multiple effector molecules essential for TCR signaling [6,7]. TCR and CD28 signaling cascades must occur concurrently and in close proximity to ensure maximal $\mathrm{T}$ cell activation [5]. Upon encounter

*Corresponding author: Kjetil Taskén, Biotechnology Centre, University of Oslo, P.O. Box 1125, N-0317 Oslo, Norway, Tel: +47-22840505; Fax: +47-22840555; E-mail: kjetil.tasken@ncmm.uio.no

Received March 13, 2013; Accepted April 16, 2013; Published April 19, 2013

Citation: Moltu K, Bjørgo E, Solstad T, Berge T, Thiede B, et al. (2013) A Proteomic Approach to Screening of Dynamic Changes in Detergent-Resistant Membranes from Activated Human Primary T Cells. J Proteomics Bioinform 6 : 072-080. doi:10.4172/jpb.1000264

Copyright: (c) 2013 Moltu K, et al. This is an open-access article distributed unde the terms of the Creative Commons Attribution License, which permits unrestricted use, distribution, and reproduction in any medium, provided the original author and source are credited. 
Citation: Moltu K, Bjørgo E, Solstad T, Berge T, Thiede B, et al. (2013) A Proteomic Approach to Screening of Dynamic Changes in DetergentResistant Membranes from Activated Human Primary T Cells. J Proteomics Bioinform 6: 072-080. doi:10.4172/jpb.1000264

with the APC, signaling molecules located to lipid rafts reorganize and cluster in proximity to the surface receptors, thus allowing signaling to occur in a rapid and efficient manner [8]. Co-stimulatory molecules participate in this process by contributing towards mobilization of proteins and remodeling of the cytoskeleton, thereby affecting lipid raft organization [9]. Both the TCR and the CD28 co-receptor have been reported to move to lipid rafts upon activation [10]. Moreover, T cell stimulation leads to further clustering of protein and lipid assemblies into larger and more stable raft domains by lipid-lipid, protein-lipid and protein-protein interactions [2], thereby concentrating key signaling molecules to favor the formation and stabilization of supramolecular complexes to promote sustained signaling upon receptor occupation $[11,12]$.

Only certain classes of proteins can associate directly with lipid rafts. Cytoplasmic proteins are recruited to rafts as a function of posttranslational modifications by covalent addition of myristoyl, palmitoyl, farnesyl or isoprenyl groups, often in specific patterns such as double acylations (e.g. for the lipid raft targeting of SFKs). Certain proteins, e.g. CD4, LAT and PAG, possess longer transmembrane domains that in combination with similar targeting signals allow association with lipid rafts whereas proteins such as CD14 and CD59 are coupled to the exoplasmic leaflet by a GPI link [13]. In resting $\mathrm{T}$ cells, lipid rafts are highly enriched in the Src kinases Lck and FynT $[4,14]$ as well as LAT [6]. Cross-linking of the TCR promotes rapid activation of these Src kinases and subsequent accumulation of tyrosine-phosphorylated substrates in lipid rafts in the early phase of TCR induction [9]. Consequently, inhibition of Lck, FynT and LAT palmitoylation has been shown to lead to their raft displacement and inhibition of $\mathrm{T}$ cell activation $[6,15,16]$.

The important role of lipid rafts in immunoreceptor signaling makes these membrane domains an interesting subject for proteomic characterization. To biochemically isolate lipid rafts, tightly packed detergent-resistant membrane (DRM) fractions can be separated from the more fluid plasma membrane components due to their resistance to solubilization in cold non-ionic detergents such as Triton X-100. This resistance allows isolation after sucrose gradient centrifugation [17], facilitating further analysis of the protein content in the lipid raft compartment. Detergent-resistance has been widely used to isolate DRM fractions as a means to study lipid rafts and their protein content, however, it is generally accepted that they do not fully correspond to native rafts under physiological conditions in size, structure or composition $[2,18]$. Numerous studies have provided information about proteins residing in DRMs from cell lines. However, it has been more challenging experimentally to obtain similar information from primary cells. In the present study, we have isolated DRMs from human primary $\mathrm{T}$ cells and performed a qualitative mass spectrometric analysis of the DRM proteome, generating a list of 425 proteins associated with DRMs. Furthermore, we have identified a group of proteins exclusively found in DRMs in either the resting or activated state of primary T cells isolated from healthy blood donors.

\section{Materials and Methods}

\section{Human peripheral $\mathrm{T}$ cell isolation and stimulation}

Buffy coats were obtained from healthy donors (Oslo University Hospital Blood Center, Oslo, Norway) and the studies were conducted under approval by the Regional Ethics Review Board. $\mathrm{CD}^{+} \mathrm{T}$ cells were purified by negative selection from buffy coats using RosetteSep T cell Enrichment Cocktail (Stem Cell Technologies, Vancouver, BC,
Canada) in accordance with the manufacturer's instructions. Purified T cells were cultured over night in a suspension of $2 \times 10^{6}$ cells per $\mathrm{ml}$ in RPMI 1640 GlutaMAX (Life Technologies, Carlsbad, CA, USA) supplemented with $10 \%$ FCS, $1 \mathrm{mM}$ sodium pyruvate, $0.9 \%(\mathrm{v} / \mathrm{v})$ MEM non-essential amino acids, $100 \mathrm{U} / \mathrm{ml}$ penicillin and $100 \mathrm{U} /$ $\mathrm{ml}$ streptomycin (all Life Technologies). To ensure maximal purity, the cells were subsequently subjected to a second isolation step with RosetteSep T cell Enrichment Cocktail. A total of approximately $1 \times 10^{9} \mathrm{~T}$ cells from four different donors were pooled together. Prior to stimulation, cells were pre-incubated at $37^{\circ} \mathrm{C}$ for $5-10 \mathrm{~min}$. Human primary $\mathrm{T}$ cells were then stimulated by first adding $0.5 \mu \mathrm{g} / \mathrm{ml}$ antiCD3 (clone OKT3) (Diatec Monoclonals AS, Oslo, Norway) and 2.5 $\mu \mathrm{g} / \mathrm{ml}$ anti-CD28 (clone 28.2)(eBioscience, San Diego, CA, USA). After $2 \mathrm{~min}, 15 \mu \mathrm{g} / \mathrm{ml} \mathrm{F}\left(\mathrm{ab}\right.$ ') ${ }_{2}$ fragment (goat anti-mouse IgG) (Jackson ImmunoResearch, West Grove, PA, USA) was added for antibody cross-ligation and stimulation lasted for the indicated time periods.

\section{Flow cytometry}

High degree of purity of the human $\mathrm{T}$ cell populations was verified by flow cytometric analysis. Cells were stained with antiCD3 conjugated with Alexa647, anti-CD19 conjugated with PerCP and anti-CD14 conjugated with FITC for $30 \mathrm{~min}$ at $4^{\circ} \mathrm{C}$ and washed with washing/staining solution ( $1 \%$ FCS, $0.09 \%$ sodium azide in PBS). Samples were run on a FACSCanto II flow cytometer (BD Biosciences, San Jose, CA, USA) equipped with $407 \mathrm{~nm}, 488 \mathrm{~nm}$ and $633 \mathrm{~nm}$ lasers. 50000 events from each sample were collected and analyzed using the FlowJo program (Tree Star Inc., Ashland, OR, USA).

\section{Isolation of detergent-resistant membrane fractions}

Isolation of Triton X-100-insoluble lipid rafts was performed as previously described [6]. Briefly, peripheral $\mathrm{T}$ cells were lysed in standard lysis buffer ( $50 \mathrm{mM}$ Hepes ( $\mathrm{pH} 7.4$ ), $10 \mathrm{mM} \mathrm{NaPPi}, 0.7 \%$ Triton $\mathrm{X}-100,50 \mathrm{mM} \mathrm{NaF}, 100 \mathrm{mM} \mathrm{NaCl}, 5 \mathrm{mM}$ EDTA, $1 \mathrm{mM}$ PMSF, $1 \mathrm{mM}$ $\mathrm{Na}_{3} \mathrm{VO}_{4}$ ) for $10 \mathrm{~min}$ on ice and lysates were subsequently homogenized completely in a Dounce homogenizer, mixed with $80 \%$ sucrose and loaded at the bottom of a $40-5 \%$ sucrose gradient in MNE-buffer (25 mM MES ( $\mathrm{pH}$ 6.5), $5 \mathrm{mM}$ EDTA, $150 \mathrm{mM} \mathrm{NaCl}$ ). Centrifugation was performed at $200000 \times \mathrm{g}$ for $20 \mathrm{~h}$ at $4^{\circ} \mathrm{C}$ using a SW55Ti rotor (Beckman Coulter Inc., Brea, CA, USA). After fractionation, all 12 fractions were tested for LAT content by immunoblotting to verify successful separation.

\section{Sample preparation for mass spectrometry}

The DRM fractions (the low-density fractions 2-5) were collected, pooled and mixed gently with ice-cold MNE-buffer and pelleted by centrifugation $(200000 \times \mathrm{g}, 1 \mathrm{~h})$. Detergent-resistant membranes were subsequently resolved in $100 \mu \mathrm{L} 100 \mathrm{mM}$ n-octyl-beta-D-glucopyranoside (OGP) followed by methanol-chloroform extraction. Briefly, $400 \mu \mathrm{L}$ methanol was added to $100 \mu \mathrm{L}$ OGP-resolved DRMs and mixed thoroughly. Next, $100 \mu \mathrm{L}$ chloroform was added, followed by addition of $300 \mu \mathrm{L}$ water and centrifugation for $1 \mathrm{~min}$ at $16,100 \times \mathrm{g}$. The top aqueous layer was removed, followed by addition of $400 \mu \mathrm{L}$ methanol and a 2 min centrifugation at 16,100 $\times$ g (Eppendorf centrifuge 5415R) (Eppendorf, Hamburg, Germany). The methanolic supernatant was removed by pipetting and the pellet dried under nitrogen prior to SDS-PAGE.

\section{SDS-PAGE}

The DRM pellet was resolved in 1X NuPAGE LDS sample buffer containing $1 \mathrm{X}$ reducing agent (Life Technologies) and heated for $5 \mathrm{~min}$ 
Citation: Moltu K, Bjørgo E, Solstad T, Berge T, Thiede B, et al. (2013) A Proteomic Approach to Screening of Dynamic Changes in DetergentResistant Membranes from Activated Human Primary T Cells. J Proteomics Bioinform 6: 072-080. doi:10.4172/jpb.1000264

at $95^{\circ} \mathrm{C}$ prior to gel electrophoresis on a $4-12 \%$ NuPAGE gel $(200 \mathrm{~V}$, $30 \mathrm{~min}$ ) (Life Technologies). Proteins were visualized by SimplyBlue SafeStain (Life Technologies).

\section{Nano-LC/LTQ-Orbitrap MS}

Bands from gels stained with SimplyBlue Safestain were excised for in-gel digestion with $0.1 \mu \mathrm{g}$ trypsin (Promega, Madison, WI, USA) in 20 $\mu \mathrm{l} 25 \mathrm{mM}$ ammonium bicarbonate, $\mathrm{pH} 7.8$ at $37^{\circ} \mathrm{C}$ for $16 \mathrm{~h}$. Each lane was cut into 14 individual bands. For each band the tryptic peptides were purified with $\mu$-C18 ZipTips (Millipore, Billerica, MA, USA) and dried using a SpeedVac Concentrator (Savant, Holbrook, NY, USA). The dried peptides were dissolved in $10 \mu \mathrm{l} \%$ formic acid in water and 5 $\mu \mathrm{l}$ were injected onto an LC/MS system consisting of a Dionex Ultimate 3000 nano-LC system (Dionex, Sunnyvale, CA, USA) connected to a linear quadrupole ion trap (LTQ)-Orbitrap mass spectrometer (Thermo Electron, Bremen, Germany) equipped with a nanoelectrospray ion source. For liquid chromatography separation we used an Acclaim PepMap 100 column (C18, $3 \mu \mathrm{m}, 100 \AA ̊)$ (Dionex) capillary of $12 \mathrm{~cm}$ bed length. The flow rate used was $300 \mathrm{nl} / \mathrm{min}$ for the nanocolumn and the solvent gradient used was 7-50\% solvent B in 40 min. Solvent A consisted of $0.1 \%$ formic acid whereas $90 \%$ aqueous acetonitrile in $0.1 \%$ formic acid was used as solvent $\mathrm{B}$. The mass spectrometer was operated in the data-dependent mode to automatically switch between OrbitrapMS and LTQ-MS/MS acquisition. Other instrument parameters were as previously described [19].

\section{Data analysis}

Raw data were processed using DTA supercharge software to generate mgf files. Afterwards, a database search was performed by tandem mass spectrometry ion search algorithms from the inhouse version of Mascot 2.2.1 (http://www.matrixscience.com) by database comparisons [20] with human entries (18117 sequences) from the UniProt database (http://www.uniprot.org). Trypsin was selected as enzyme with a maximum of two missed cleavage sites and tolerance of $5 \mathrm{ppm}$ for the precursor ion and 0.5 Da for the MS/MS fragments was applied. Moreover, methionine oxidation, acetylation at protein $\mathrm{N}$-terminus, deamidation of asparagines and glutamines and propionamide formation of cysteines were allowed as variable modifications. Proteins were considered identified by Mascot if the following criteria were achieved for at least one peptide: probability $<0.05$ and a Mascot ion score above 30. Gene Ontology (GO) annotation pie charts based on the complete list of UniProt Knowledgebase accession entries were generated from STRAP (Software Tool for Researching Annotations of Proteins) (http://www.bumc.bu.edu/ cardiovascularproteomics/cpctools/strap) [21]. Interaction network analysis of known and predicted protein interactions were generated by STRING (Search Tool for the Retrieval of Interacting Genes/ Proteins) (http://string-db.org) [22] based on 'Neighborhood', 'Gene fusion', 'Co-occurrence', 'Experiments', 'Databases', 'Text mining' and 'Homology' evidence sources. STRING gives scores from 0.001 to 0.999 and in our initial network analysis of the 425 proteins, the highest (0.900) level of confidence was chosen, while high confidence (0.700) was chosen for the network display of the 190 interacting proteins closest associated with the TCR. The GO term enrichment in this target set of 190 proteins was compared to a background set of 972 proteins identified in $\mathrm{T}$ cells from human peripheral blood constituents by Haudek et al. [23] by a GOrilla analysis (http://cblgorilla.cs.technion.ac.il) [24]. GOrilla searches for enriched GO terms in two unranked lists of genes, one target and one background set, using standard hypergeometric statistics. The resulting enriched GO terms are visualized using a DAG graphical representation with color coding reflecting their degree of enrichment. Enrichment is defined as $(\mathrm{b} / \mathrm{n}) /(\mathrm{B} / \mathrm{N})$ where $\mathrm{b}$ is the number of genes in the target set that are associated with a specific GO term, $\mathrm{n}$ is the number of genes in the target set (190), B is the total number of genes associated with a specific GO term (1021) and N is the total number of genes (1035). Graphs of experimentally determined (both low- and high-throughput data) posttranslational modifications (PTMs) for the target set of 190 proteins were generated using PhospoSitePlus (http://www.phosphosite.org) [25]. 2249 experimentally verified interactions between the 425 proteins were found with the InnateDB database (http://www.innatedb.ca) [26], and the interaction search was visualized using subcellular localization annotation. Layout was generated by Cytoscape 2.8.2 with Cerebral plugin.

\section{Western blot analysis}

Lysates were separated by SDS-PAGE and transferred to polyvinylidene fluoride membranes (PVDF) (Immobilon-P, Millipore, Oslo, Norway) by electroblotting. Membranes were subsequently blocked in 5\% non-fat dry milk in TBS-T $(0.1 \%$ Tween 20 in TBS) at room temperature for $1 \mathrm{~h}$, followed by incubation at $4^{\circ} \mathrm{C}$ over night with the indicated primary antibodies. Visualization was achieved using horseradish peroxidase (HRP)-conjugated secondary antibodies (Jackson ImmunoResearch), SuperSignal West Pico Chemiluminescent Substrate (Pierce, Rockford, IL, USA) and ECL Detection Reagents (GE Healthcare Lifesciences, Little Chalfont, UK). Primary antibodies used were anti-Csk (Santa Cruz Biotechnology, Santa Cruz, CA, USA), anti-ILF3 (Abcam, Cambridge, UK), anti-integrin alpha-L/CD11a (Abcam), anti-LAT (Upstate Biotechnology, Lake Placid, NY, USA), anti-SH3KBP1/CIN85 (Sigma-Aldrich, St. Louis, MO, USA) and antiZAP-70 (BD Biosciences).

\section{Results and Discussion}

\section{Isolation of DRMs from human primary $\mathrm{T}$ cells}

In the present study, we have examined the protein content in DRMs of resting versus stimulated human primary $\mathrm{T}$ cells as shown schematically in figure 1A. Cells were isolated from healthy blood donors with a purity of approximately $90 \%$ or higher, as evaluated by flow cytometry (Figure $1 \mathrm{~B}$ ), and subjected to either CD3/CD28 costimulation or left unstimulated. Subsequently, cell lysis was performed at $4^{\circ} \mathrm{C}$ in the presence of $0.7 \%$ Triton X-100 followed by discontinuous sucrose-density gradient ultracentrifugation. Cholesterol-enriched DRMs have previously been shown to be present in the low-density fractions and this was also confirmed in the present study, using the DRM-associated protein LAT as a marker (Figure 1C). In order to increase the purity of the DRM fractions, peak raft fractions (fractions 2-5) were pooled and subjected to methanol-chloroform precipitation to remove interfering lipids [19].

\section{Qualitative analysis of the DRM proteome}

T cells can initiate intracellular signaling responses remarkably fast. LAT phosphorylation and calcium flux occur within seconds of TCR stimulation and cytoskeletal polarization takes place within minutes [27]. The highly dynamic lipid raft structures allow rapid diffusion of their constituent molecules over a large area [28], thus co-localizing the TCRs and several other signaling proteins. Here, we examined the dynamics of protein association with DRMs in response to TCR/ CD28 engagement for three minutes. Qualitative analysis of the protein content in DRMs from either resting or stimulated primary $\mathrm{T}$ cells 
A

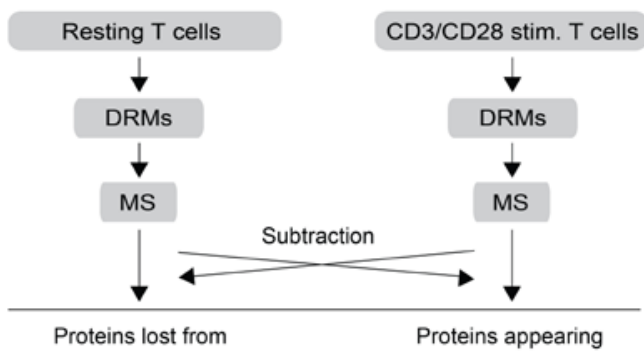

DRMs upon activation

in DRMs upon activation

B

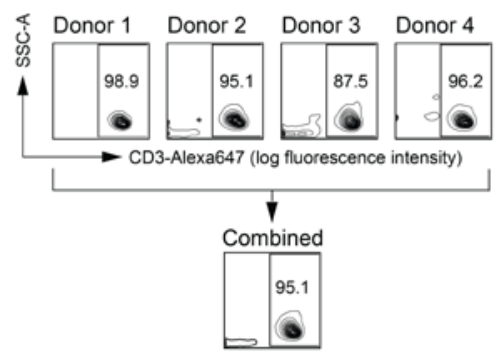

C

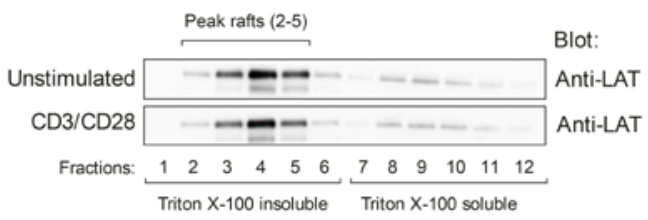

Figure 1: Experimental setup and preparation of detergent-resistant membranes. (A) Primary $\mathrm{CD} 3^{+} \mathrm{T}$ cells from four healthy donors were pooled together and left resting or stimulated (stim.) with anti-CD3 and anti-CD28 for two minutes followed by cross-ligation with $F\left(a b{ }_{2}\right)_{2}$-fragments for three minutes. After lysis on ice using $0.7 \%$ Triton X-100, detergent-resistant membranes (DRMs) were isolated by sucrose gradient ultracentrifugation at $4^{\circ} \mathrm{C}$. The ultra-light fractions (fractions $2-5$ ) were pooled and lipids removed by chloroform-methanol extraction. Precipitated proteins were subsequently resolved in sample buffer and separated by SDS-PAGE gel electrophoresis. Whole gel lanes were digested with trypsin and analyzed by Nano-LC/LTQOrbitrap MS. After a qualitative mass spectrometric analysis, a set of proteins found to be present solely in either DRMs of resting T cells or in DRMs of CD3/ CD28 co-stimulated T cells was identified. (B) One representative experiment using flow cytometry, showing the purity of primary $T$ cells isolated from each of the four blood donors and after pooling of the four populations. (C) Isolation of DRMs (fractions 2-5) was verified by the content of the transmembrane protein LAT (constitutively associated with DRMs).

was performed in three individual experiments, and only proteins identified in two out of three separate experiments were included in the final analysis.

Altogether, the Mascot database search identified 425 proteins associated with DRMs from human primary T cells (Supplementary Table 1) with a false discovery rate below $2 \%$. For a global overview of the T cell DRM proteome, the proteins were first categorized according to the three main Gene Ontology (GO) project domains: 'cellular components', 'biological processes' and 'molecular function' as annotated by STRAP (Software Tool for Researching Annotations of Proteins). The results were summarized in pie charts as shown in figure 2. Altogether, the 425 proteins were identified with 1020 unique GO terms classifying 'cellular components'. $17.6 \%$ of these counts were linked to the GO term 'cytoplasm' and $13.9 \%$ of the counts were linked to the term 'plasma membrane' (Figure 2, left). Terms linked to intracellular organelles such as 'mitochondria', 'nucleus' and 'endoplasmic reticulum' (ER) also acquired many counts, 5.8, 13.6 and $4.6 \%$ respectively. Based on previous biochemical isolations, DRMs may also be found in compartments other than the plasma membrane [17]. Lipid raft components may move laterally within the plasma membrane as well as traffic continuously between the plasma membrane and internal compartments [29]. In addition, specific raft lipids and raft proteins are synthesized in the ER/Golgi before being transported to the plasma membrane [30]. Thus, many proteins important for protein synthesis and vesicle trafficking were therefore, as expected, identified in the DRM fractions.

It has been discussed whether mitochondria contribute to the signaling complex that forms at the interface between the APC and the T cell [31,32]. Most proteomic investigations involving DRMs report mitochondrial proteins to be present in the preparations, in particular mitochondrial adenosine triphosphate (ATP) synthase subunits and the voltage-dependent anion selective channels (VDACs). Quintana et al. [31] report that translocation of mitochondria to the plasma membrane is required to maintain calcium-influx across the plasma membrane. Furthermore, expression of the mitochondrial ATP synthase complex has been demonstrated on the surface of the plasma membrane [33]. However, mitochondrial proteins in DRMs are not sensitive to raft disruption by the cholesterol chelating agent methyl-beta-cyclodextrin, consequently rejecting the notion that mitochondrial proteins reside in lipid rafts [32]. Thus, it is likely that some of the proteins included in the present study are located in other membrane compartments of the cell.

The TCR signaling cascade is tightly integrated with cytoskeletal proteins and a large fraction ( $8.6 \%$ of the counts) of the detected DRM proteins was found to be associated with the cytoskeleton (Figure 2, left). The cytoskeleton consists of filament-forming proteins such as actin, myosin II and tubulin. These filaments are coordinated by the polarity network and also directly through receptor signaling. Integrindependent adhesion and TCR signaling require F-actin and are partly dependent on myosin II [34]. In the GO domain 'biological processes', the largest groups were proteins categorized by their involvement in 'regulation' and 'cellular processes' (53.9\% of the counts) (Figure 2 , middle). Finally, within the domain 'molecular function', more than $50 \%$ of the GO terms were associated with 'binding' and $23.7 \%$ were associated with 'catalytic activity' (Figure 2, right). The list of proteins include $\mathrm{T}$ cell receptor binding proteins, cytoskeletal proteins, transmembrane receptors, cell surface glycoproteins, kinase binding proteins and trafficking proteins, all groups of proteins likely to participate in various aspects of $\mathrm{T}$ cell function.

After comparison of the two datasets from resting and stimulated T cells, we found 16 proteins exclusively present in DRMs of resting T cells (Table 1) whereas 19 proteins were exclusively observed in DRMs of stimulated T cells (Table 2). Thus, the protein content of DRMs was not significantly altered by TCR engagement. However, quantitative changes in protein levels are not covered by this approach and are also of functional importance in signal transduction. Quantitative proteomic approaches dissecting antigen receptor-mediated dynamics of DRMs have been reported $[11,35,36]$. However, these studies were performed on material obtained from cell lines and have been based mostly on metabolic labeling of cells by differential feeding with light or heavy isotopic amino acids, an approach which is not possible with primary cells. Furthermore, there is no consensus on what proportion of each protein is needed to be detergent-insoluble for a protein to be categorized as lipid raft-associated. Consequently, examples provided 
Citation: Moltu K, Bjørgo E, Solstad T, Berge T, Thiede B, et al. (2013) A Proteomic Approach to Screening of Dynamic Changes in DetergentResistant Membranes from Activated Human Primary T Cells. J Proteomics Bioinform 6: 072-080. doi:10.4172/jpb.1000264
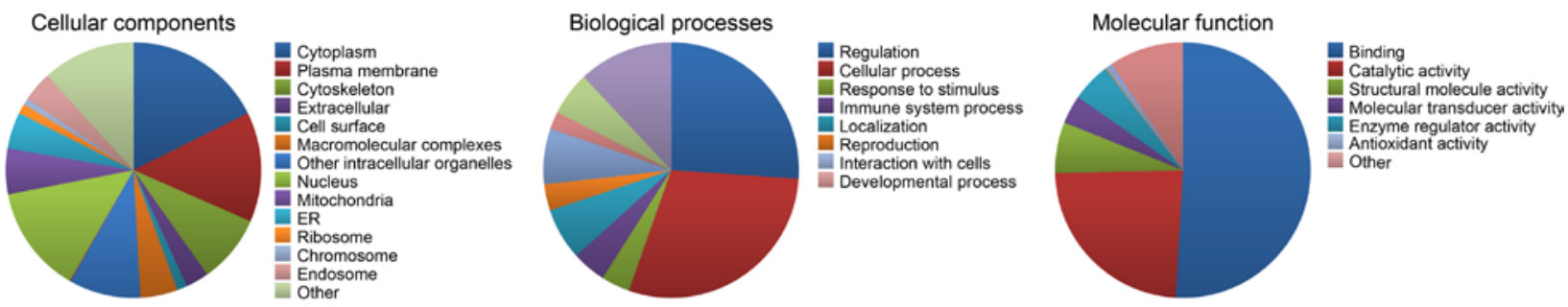

Figure 2: Graphical representation of the GO term association data for the 425 proteins identified in DRMs of human primary T cells as annotated by STRAP. Identified proteins were grouped into pie charts according to the three main GO categories 'cellular components', 'biological processes' and 'molecular function' as indicated, and each pie slice was labeled with the GO subcategory.

\begin{tabular}{|c|c|c|c|c|}
\hline Identified proteins & UniprotKBacc. entry & Mascot score & Th. $M_{w}(\mathrm{Da})$ & $\begin{array}{l}\text { \# unique } \\
\text { peptides }\end{array}$ \\
\hline HLA class I histocompatibility antigen, A-3 alpha chain & 1A03_HUMAN & 408 & 40815 & 3 \\
\hline HLA class I histocompatibility antigen, B-44 alpha chain & 1B44_HUMAN & 188 & 40456 & 2 \\
\hline HLA class I histocompatibility antigen, $\mathrm{Cw}-12$ alpha chain & 1C12_HUMAN & 428 & 40860 & 3 \\
\hline Apolipoprotein O & APOO_HUMAN & 66 & 22271 & 2 \\
\hline Serine-protein kinase ATM & ATM_HUMAN & 39 & 350419 & 2 \\
\hline ATP synthase subunit e, mitochondrial & ATP5I_HUMAN & 54 & 7928 & 3 \\
\hline B-cell receptor-associated protein 31 & BAP31_HUMAN & 82 & 27974 & 2 \\
\hline Apoptosis regulator $\mathrm{Bcl}-2$ & BCL2_HUMAN & 46 & 26249 & 2 \\
\hline Cdc42 smalleffector protein 2 & C42S2_HUMAN & 47 & 9217 & 2 \\
\hline Glutathione S-transferase kappa 1 & GSTK1_HUMAN & 89 & 25480 & 2 \\
\hline 3-hydroxyacyl-CoA dehydrogenase type-2 & HCD2_HUMAN & 73 & 26906 & 2 \\
\hline Intercellularadhesionmolecule 3 & ICAM3_HUMAN & 52 & 59503 & 2 \\
\hline Integrin alpha-L/CD11a & ITAL_HUMAN & 72 & 128688 & 5 \\
\hline Multidrugresistance protein 1 & MDR1_HUMAN & 136 & 141373 & 4 \\
\hline Microsomalglutathione S-transferase 3 & MGST3_HUMAN & 66 & 16506 & 2 \\
\hline Torsin-1A-interacting protein 1 & TOIP1_HUMAN & 85 & 66208 & 3 \\
\hline
\end{tabular}

Table 1: Proteins observed to be exclusively present in DRMs of resting $\mathrm{CD3}^{+} \mathrm{T}$ cells. The table lists all proteins solely observed in DRMs from resting human primary T cells including their UniProt Knowledgebase accession entry, total Mascot score, theoretical molecular mass (Th. $\mathrm{M}_{\mathrm{w}}$ ) and number (\#) of unique peptides identified. The table represents combined data from three separate experiments and all proteins had to be identified in at least two individual experiments in order to be included.

\begin{tabular}{|l|l|c|c|}
\hline Identified proteins & UniprotKBacc. entry & Mascot score & $\begin{array}{c}\text { Th. M } \\
\text { w }\end{array}$ (Da) \\
\hline AFG3-like protein 2 & \# unique \\
peptides
\end{tabular}

Table 2: Proteins observed to be exclusively present in DRMs of stimulated $\mathrm{CD3}^{+} \mathrm{T}$ cells. The table lists all proteins solely observed in DRMs from human primary T cells upon CD3/CD28 co-stimulation including their UniProt Knowledgebase accession entry, total Mascot score, theoretical molecular mass (Th. $\mathrm{M}_{\mathrm{w}}$ ) and number (\#) of unique peptides identified. The table represents combined data from three separate experiments and all proteins had to be identified in at least two individual experiments in order to be included. 
Citation: Moltu K, Bjørgo E, Solstad T, Berge T, Thiede B, et al. (2013) A Proteomic Approach to Screening of Dynamic Changes in DetergentResistant Membranes from Activated Human Primary T Cells. J Proteomics Bioinform 6: 072-080. doi:10.4172/jpb.1000264

in the literature define cut-offs for lipid raft-associated proteins in the range of $10-50 \%$ of the total protein content [18]. Quantitative studies are therefore not unambiguous in this respect.

\section{Rearrangement of DRMs upon T cell activation}

As revealed by table 1, the major histocompatibility complex (MHC) molecules (also known as human leukocyte antigen (HLA)) appear to be displaced from the exoplasmatic side of the T cell membrane upon TCR stimulation. This is most likely a result of recycling or degradation of these proteins post $\mathrm{T}$ cell activation. Furthermore, both integrin alpha-L/CD11a and the intercellular adhesion molecule (ICAM)-3 were uniquely identified in DRMs of resting T cells (Table 1). ICAMs mediate leukocyte migration into tissues and antigen-presentation to $\mathrm{T}$ cells. Triggering of the TCR/CD3 complex induces intracellular signals that activate integrin alpha-L/CD11a and uncouples the surface receptor from the actin cytoskeleton, resulting in receptor clustering and enhanced binding to ICAM [37]. This serves as an example of how movement of a receptor over the cell surface is prevented through linkage to cytoskeletal elements in the resting state of the cell, thereby inhibiting clustering and strong ligand binding.

Cell division cycle 42 (Cdc42), a member of the mammalian Rho GTPase family, was only found in DRMs isolated from stimulated $\mathrm{T}$ cells (Table 2). Regulation of the Rho GTPases is complex, but consensus is that efficient Rho GTPase signaling requires an association with the lipophilic membrane. Furthermore, Cdc42 small effector protein 2 acts downstream of Cdc42, induces actin filament assembly and has previously been observed to be recruited to the TCR prior to actin polymerization [38]. In this study, Cdc42 small effector protein 2 was identified only in DRMs of resting T cells (Table 1).

Interleukin enhancer-binding factor 3 (ILF3) is a positive transcriptional regulator observed in DRMs of stimulated T cells (Table 2). ILF3 localizes to both cytosol and nucleus, but proteomic examinations of the $\mathrm{T}$ cell nucleolus has recently revealed that its nuclear localization is not constitutive as less than $35 \%$ of the cells display ILF3 in the nucleolus [39]. Recruitment of the Cbl-binding protein CIN85 (also known as SH3 domain-containing kinase-binding protein 1) in the stimulated fractions (Table 2) is also noteworthy. CIN85 is a multiadaptor protein involved in different cellular functions, including down-regulation of activated receptor tyrosine kinases [40].

Western blot analysis was used to verify DRM association for a selection of proteins found in tables 1 and 2 (Figure 3). This confirmed recruitment of CIN85 and ILF3 to DRMs upon T cell activation as well as loss of integrin alpha-L/CD11a. Well-known lipid raft-associated proteins and proteins shown to be regulated upon TCR triggering were included as markers. The non-receptor tyrosine kinase ZAP-70 was used as a positive control for recruitment to DRMs upon T cell stimulation. ZAP-70 is recruited to the membrane within seconds after activation and the activation-induced redistribution of ZAP-70 is of long duration [41]. The negative regulator C-terminal Src kinase (Csk) was used as a marker for displacement from DRMs upon T cell activation. In resting T cells, Csk is constitutively localized in DRMs through docking to the hyperphosphorylated transmembrane adaptor protein PAG $[42,43]$. However, upon T cell activation, PAG is rapidly dephosphorylated and Csk is transiently displaced to the cytosol until Fyn-mediated phosphorylation of PAG results in re-recruitment of Csk to DRMs [42,44]. Furthermore, the palmitoylated transmembrane adaptor protein LAT was used as a loading control since this protein is constitutively present in T cell DRMs. LAT has a very short extracellular

\section{Peak raft fractions $(2-5)$}

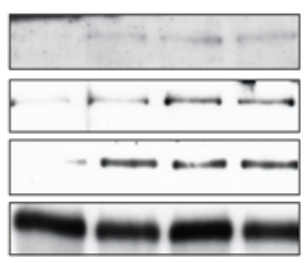

Blot:

Anti-ILF3

Anti-CIN85

Anti-ZAP-70

Anti-LAT

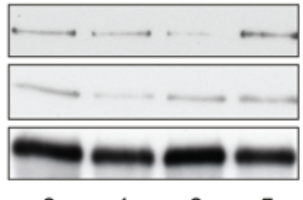

Anti-CD11a

Anti-Csk

Anti-LAT

\begin{tabular}{cccc}
0 & 1 & 3 & 5 \\
\hline Anti-CD3/CD28+F(ab')
\end{tabular}

$\min$

Figure 3: Recruitment or displacement of proteins from DRMs upon CD3/CD28 co-stimulation. To confirm some of the findings in tables 1 and 2 , human primary $T$ cells were stimulated and DRMs isolated as described in figure 1. Peak raft fractions (combined fractions 2-5) were subsequently subjected to immunoblotting and analyzed for the indicated protein content. Proteins like ILF3 and CIN85 were recruited to the DRMs upon CD3/CD28 triggering (upper panel) whereas integrin 1-alpha/CD11a was displaced from the DRMs upon co-stimulation (lower panel). ZAP-70 and Csk were used as positive controls of recruitment or displacement, respectively. LAT was used as loading control as it constitutively resides in the DRMs of T cells.

domain and a long cytoplasmic domain that contains multiple phosphorylation sites which function as docking sites for a diversity of signaling molecules [45]. Anti-LAT immunoblotting verified that comparable protein amounts were loaded on the SDS-PAGE gel from each peak raft fraction (combined fractions 2-5) (Figure 3).

\section{Bioinformatic characterization of the DRM proteome}

Lipid raft structures provide a unique environment for stabilizing protein interactions. We therefore performed a STRING (Search Tool for the Retrieval of Interacting Genes/Proteins) analysis of the proteins identified in DRMs of both resting and activated T cells to visualize a protein network. STRING integrates experimental and predicted interactions, and the interactions are provided with a confidence score ranging from low (0.150) to highest (0.900) confidence. A selection of 190 proteins in DRMs with the closest association to members of the TCR/CD3 complex was generated based on the highest confidence level (0.900) (Supplementary Table 2). The interconnections in this selected subgroup (Supplementary Figure 1) indicate that proteomic analysis of DRMs does indeed identify interacting proteins in the TCR proximal environment. In addition, using the gene ontology enrichment analysis and visualization tool GOrilla, we compared the selected subgroup of 190 proteins to a background set of 972 proteins identified in T cells from human peripheral blood constituents [23] (Figure 4). Altogether, our findings suggest that a major subset of the DRM proteins in T cells has a predominant role in cytoskeleton function and rearrangement whereas other significant subsets are involved in protein interactions and $\mathrm{T}$ cell signaling. Integrating available experimental evidence from curated databases on the proteins in the target set of 190 proteins revealed that the majority of these have a high percentage of posttranslational modifications (Figure 5), the most prominent being phosphorylation on tyrosine, serine or threonine residues. In addition, we also observed that proteins exclusively identified in DRM fractions from stimulated $\mathrm{T}$ cells appear to have a higher degree of ubiquitination 


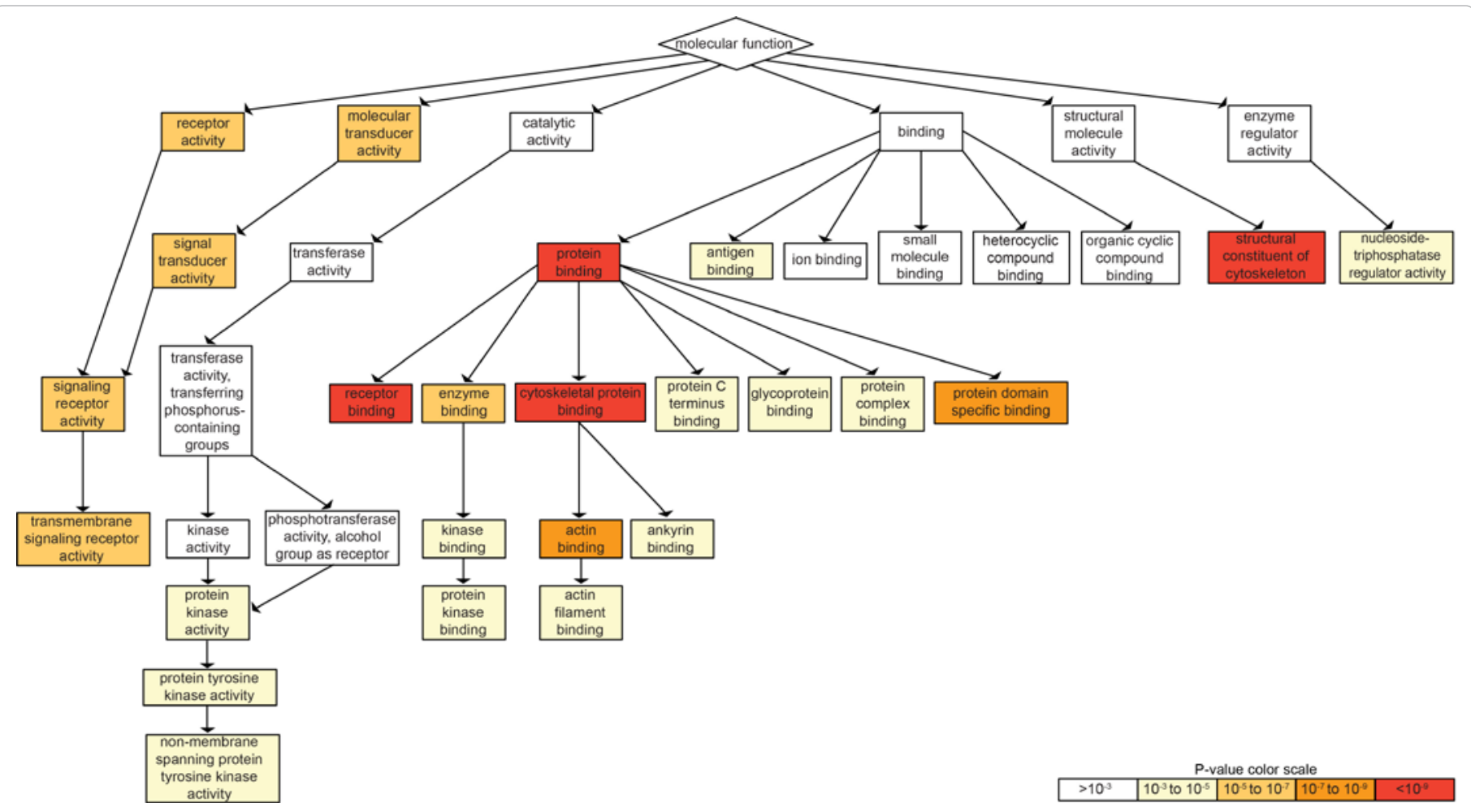

Figure 4: GO term enrichment analysis according to the 'molecular function' ontology.

A target set of 190 proteins, closely associated with the T cell receptor was identified and compared to a background set of 972 proteins identified in T cells from human peripheral blood constituents [23], and data were visualized using the software GOrilla. GOrilla searches for enriched GO terms in two unranked lists of genes, the target and background set. The enrichment p-values are calculated using standard hyper geometric statistics. Graphics were modified from GOrilla.

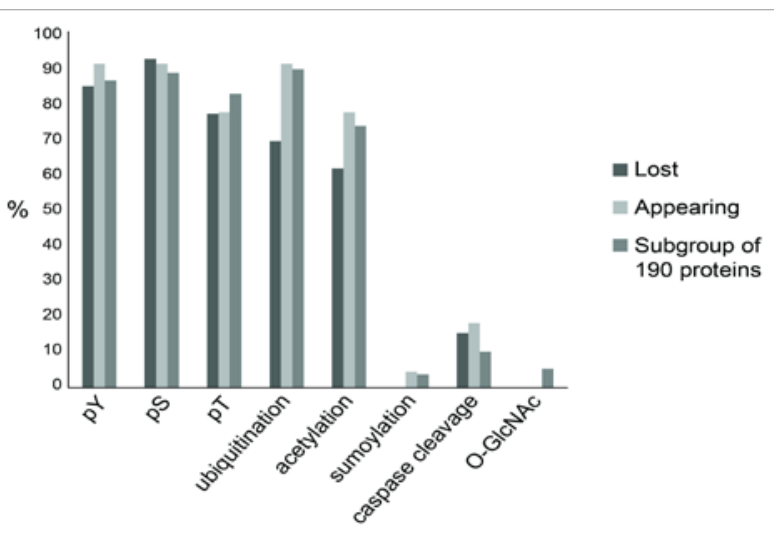

Figure 5: Common post-translational modifications (PTMs) of the proteins exclusively lost from or appearing in DRMs upon T cell activation as well as PTMs for the subgroup of 190 proteins shown to be closely associated with the T cell receptor.

Experimentally observed PTMs for the selected proteins were found using the database PhosphoSitePlus.

Abbreviations: $\mathrm{pY} / \mathrm{S} / \mathrm{T}$, phosphorylation on tyrosine/serine/threonine residues; O-GlcNAc, O-linked $\mathrm{N}$-acetylglucosamine.

reported than proteins lost upon stimulation, which may indicate that they are under tighter regulatory control.

Finally, 2249 experimentally verified interactions between the 425 proteins observed in DRMs were identified using the InnateDB database. Figure 6 demonstrates how interactions in the whole dataset of 425 proteins are arranged into pathways sorted by their reported subcellular location. Proteins identified in DRMs may extend beyond the lipid bilayer into the extracellular environment, associate with the membrane by lipid anchoring or by binding to other membrane proteins. Proteins may also locate mainly to compartments such as the cytoplasm and the nucleus, but appear in the DRMs due to constant shuffling of proteins from the external to the internal environment of the cell. Subunits of protein kinase A (PKA, gene name PRKAR1A) stand out as a central junction in this presentation of protein networks, substantiating the vital role PKA has in regulating a number of cellular processes important for immune activation and control. PKA has been demonstrated to regulate antigen receptor-induced signaling both by altering protein-protein interactions and by changing the enzymatic activity of target proteins [46].

Cell activation-dependent changes in the size and distribution of lipid rafts are under intense investigation. It has been argued that detergent-resistance as a means of isolating lipid raft proteins is an artificial, unstable and condition-variable approach that in itself could induce the formation of membrane domains, thus not representing physiologically relevant information $[2,18]$. Results regarding function and composition of lipid raft fractions will undoubtedly vary with the methods and conditions used, both during isolation and protein analysis. However, so far data demonstrate that Triton X-100 does not induce domain formation, although detergent-treatment may increase domain size by coalescing pre-existing nanodomains [47]. The ongoing controversy to which extent DRMs correspond to in vivo lipid rafts will hopefully be clarified with new super-resolution technologies. 
Citation: Moltu K, Bjørgo E, Solstad T, Berge T, Thiede B, et al. (2013) A Proteomic Approach to Screening of Dynamic Changes in DetergentResistant Membranes from Activated Human Primary T Cells. J Proteomics Bioinform 6: 072-080. doi:10.4172/jpb.1000264

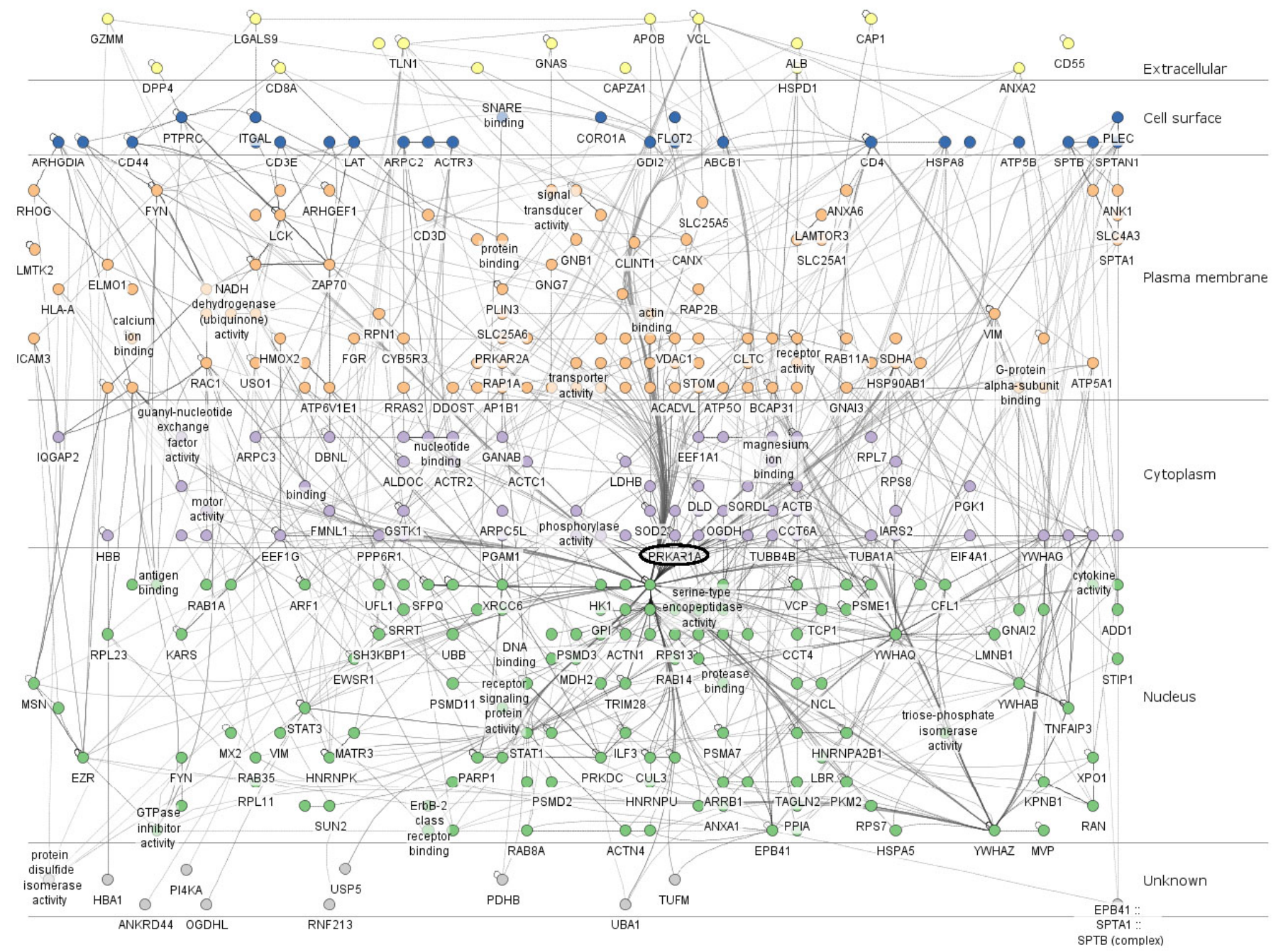

Figure 6: Overview of the interactions between the 425 proteins identified in DRMs as generated by the InnateDB database.

2249 experimentally verified interactions based on one or more publications were identified and then visualized using subcellular localization annotation in InnateDB The layout was generated by Cytoscape with cerebral plugin.

\section{Conclusion}

Our qualititative proteomic approach to analysis of proteins in $\mathrm{T}$ cell lipid rafts has identified more than 400 proteins in DRMs, some of which exit or enter upon $\mathrm{T}$ cell activation. A majority of the identified proteins can be mapped onto T cell signaling pathways, supporting the idea of lipid rafts as signaling platforms.

\section{Acknowledgements}

We are grateful to Magnus Arntzen, Dr. Ian Donaldson and Julio SaezRodrigues for helpful discussions of the data. This work was funded by grants from the Research Council of Norway, Molecular Life Science, UiO and Norwegian Cancer Society.

\section{References}

1. Simons K, Ikonen E (1997) Functional rafts in cell membranes. Nature 387: 569-572.

2. Simons K, Gerl MJ (2010) Revitalizing membrane rafts: new tools and insights. Nat Rev Mol Cell Biol 11: 688-699.

3. Horejsí V (2005) Lipid rafts and their roles in T-cell activation. Microbes Infect 7: 310-316.

4. Xavier R, Brennan T, Li Q, McCormack C, Seed B (1998) Membrane compartmentation is required for efficient $\mathrm{T}$ cell activation. Immunity 8: 723 732.

5. Acuto O, Michel F (2003) CD28-mediated co-stimulation: a quantitative support for TCR signalling. Nat Rev Immunol 3: 939-951.

6. Zhang W, Trible RP, Samelson LE (1998) LAT palmitoylation: its essential role in membrane microdomain targeting and tyrosine phosphorylation during $\mathrm{T}$ cell activation. Immunity 9: 239-246.

7. Mustelin T, Taskén K (2003) Positive and negative regulation of T-cell activation through kinases and phosphatases. Biochem J 371: 15-27.

8. Hunter T (2000) Signaling--2000 and beyond. Cell 100: 113-127.

9. Alonso MA, Millán J (2001) The role of lipid rafts in signalling and membrane trafficking in T lymphocytes. J Cell Sci 114: 3957-3965.

10. Sadra A, Cinek T, Imboden JB (2004) Translocation of CD28 to lipid rafts and costimulation of IL-2. Proc Natl Acad Sci U S A 101: 11422-11427.

11. Gupta N, Wollscheid B, Watts JD, Scheer B, Aebersold R, et al. (2006) Quantitative proteomic analysis of $B$ cell lipid rafts reveals that ezrin regulates antigen receptor-mediated lipid raft dynamics. Nat Immunol 7: 625-633.

12. Langlet $C$, Bernard AM, Drevot $P, H e H T$ (2000) Membrane rafts and signaling by the multichain immune recognition receptors. Curr Opin Immunol 12: 250255. 
Citation: Moltu K, Bjørgo E, Solstad T, Berge T, Thiede B, et al. (2013) A Proteomic Approach to Screening of Dynamic Changes in DetergentResistant Membranes from Activated Human Primary T Cells. J Proteomics Bioinform 6: 072-080. doi:10.4172/jpb.1000264

13. Mumby SM (1997) Reversible palmitoylation of signaling proteins. Curr Opin Cell Biol 9: 148-154.

14. Montixi C, Langlet C, Bernard AM, Thimonier J, Dubois C, et al. (1998) Engagement of $\mathrm{T}$ cell receptor triggers its recruitment to low-density detergentinsoluble membrane domains. EMBO J 17: 5334-5348.

15. Webb Y, Hermida-Matsumoto L, Resh MD (2000) Inhibition of protein palmitoylation, raft localization, and $\mathrm{T}$ cell signaling by 2-bromopalmitate and polyunsaturated fatty acids. J Biol Chem $275: 261-270$.

16. Kabouridis PS, Magee Al, Ley SC (1997) S-acylation of LCK protein tyrosine kinase is essential for its signalling function in T lymphocytes. EMBO $\mathrm{J} 16$ : 4983-4998.

17. Brown DA, Rose JK (1992) Sorting of GPI-anchored proteins to glycolipidenriched membrane subdomains during transport to the apical cell surface. Cell 68: 533-544

18. Shaw AS (2006) Lipid rafts: now you see them, now you don't. Nat Immunol 7: 1139-1142.

19. Solstad T, Bjørgo E, Koehler CJ, Strozynski M, Torgersen KM, et al. (2010) Quantitative proteome analysis of detergent-resistant membranes identifies the differential regulation of protein kinase $\mathrm{C}$ isoforms in apoptotic $\mathrm{T}$ cells. Proteomics 10: 2758-2768.

20. Perkins DN, Pappin DJ, Creasy DM, Cottrell JS (1999) Probability-based protein identification by searching sequence databases using mass spectrometry data. Electrophoresis 20: 3551-3567.

21. Bhatia VN, Perlman DH, Costello CE, McComb ME (2009) Software tool for researching annotations of proteins: open-source protein annotation software with data visualization. Anal Chem 81: 9819-9823.

22. Szklarczyk D, Franceschini A, Kuhn M, Simonovic M, Roth A, et al. (2011) The STRING database in 2011: functional interaction networks of proteins, globally integrated and scored. Nucleic Acids Res 39: D561-568.

23. Haudek VJ, Slany A, Gundacker NC, Wimmer H, Drach J, et al. (2009) Proteome maps of the main human peripheral blood constituents. J Proteome Res 8: 3834-3843.

24. Eden E, Navon R, Steinfeld I, Lipson D, Yakhini Z (2009) GOrilla: a tool for discovery and visualization of enriched GO terms in ranked gene lists. BMC Bioinformatics 10: 48

25. Hornbeck PV, Kornhauser JM, Tkachev S, Zhang B, Skrzypek E, et al. (2012) PhosphoSitePlus: a comprehensive resource for investigating the structure and function of experimentally determined post-translational modifications in man and mouse. Nucleic Acids Res 40: D261-270.

26. Breuer K, Foroushani AK, Laird MR, Chen C, Sribnaia A, et al. (2013) InnateDB systems biology of innate immunity and beyond--recent updates and continuing curation. Nucleic Acids Res 41: D1228-1233.

27. Huse M, Klein LO, Girvin AT, Faraj JM, Li QJ, et al. (2007) Spatial and tempora dynamics of $\mathrm{T}$ cell receptor signaling with a photoactivatable agonist. Immunity 27: 76-88.

28. Kusumi A, Koyama-Honda I, Suzuki K (2004) Molecular dynamics and interactions for creation of stimulation-induced stabilized rafts from small unstable steady-state rafts. Traffic 5: 213-230.

29. Nichols BJ, Kenworthy AK, Polishchuk RS, Lodge R, Roberts TH, et al. (2001) Rapid cycling of lipid raft markers between the cell surface and Golgi complex. J Cell Biol 153: 529-541.

30. Foster LJ, De Hoog CL, Mann M (2003) Unbiased quantitative proteomics of lipid rafts reveals high specificity for signaling factors. Proc Natl Acad Sci U S A 100: 5813-5818.

31. Quintana A, Schwindling C, Wenning AS, Becherer U, Rettig J, et al. (2007) $\mathrm{T}$ cell activation requires mitochondrial translocation to the immunological synapse. Proc Natl Acad Sci U S A 104: 14418-14423.

32. Zheng YZ, Berg KB, Foster LJ (2009) Mitochondria do not contain lipid rafts, and lipid rafts do not contain mitochondrial proteins. J Lipid Res 50: 988-998.

Citation: Moltu K, Bjørgo E, Solstad T, Berge T, Thiede B, et al. (2013) A Proteomic Approach to Screening of Dynamic Changes in Detergent-Resistant Membranes from Activated Human Primary T Cells. J Proteomics Bioinform 6: 072-080. doi:10.4172/jpb.1000264
33. Bae TJ, Kim MS, Kim JW, Kim BW, Choo HJ, et al. (2004) Lipid raft proteome reveals ATP synthase complex in the cell surface. Proteomics 4: 3536-3548.

34. Dustin ML, Depoil D (2011) New insights into the T cell synapse from single molecule techniques. Nat Rev Immunol 11: 672-684.

35. Bini L, Pacini S, Liberatori S, Valensin S, Pellegrini M, et al. (2003) Extensive temporally regulated reorganization of the lipid raft proteome following T-cel antigen receptor triggering. Biochem J 369: 301-309.

36. Lin SL, Chien CW, Han CL, Chen ES, Kao SH, et al. (2010) Temporal proteomics profiling of lipid rafts in CCR6-activated T cells reveals the integration of actin cytoskeleton dynamics. J Proteome Res 9: 283-297.

37. Lub M, van Kooyk Y, van Vliet SJ, Figdor CG (1997) Dual role of the actin cytoskeleton in regulating cell adhesion mediated by the integrin lymphocyte function-associated molecule-1. Mol Biol Cell 8: 341-351.

38. Pirone DM, Fukuhara S, Gutkind JS, Burbelo PD (2000) SPECs, small binding proteins for Cdc42. J Biol Chem 275: 22650-22656.

39. Jarboui MA, Wynne K, Elia G, Hall WW, Gautier VW (2011) Proteomic profiling of the human T-cell nucleolus. Mol Immunol 49: 441-452.

40. Dikic I (2002) CIN85/CMS family of adaptor molecules. FEBS Lett 529: 110 115

41. Sloan-Lancaster J, Presley J, Ellenberg J, Yamazaki T, Lippincott-Schwartz $\mathrm{J}$, et al. (1998) ZAP-70 association with T cell receptor zeta (TCRzeta) fluorescence imaging of dynamic changes upon cellular stimulation. J Cell Bio 143: 613-624.

42. Brdicka T, Pavlistová D, Leo A, Bruyns E, Korínek V, et al. (2000) Phosphoprotein associated with glycosphingolipid-enriched microdomains (PAG), a novel ubiquitously expressed transmembrane adaptor protein, binds the protein tyrosine kinase csk and is involved in regulation of $\mathrm{T}$ cell activation. J Exp Med 191: 1591-1604

43. Kawabuchi M, Satomi Y, Takao T, Shimonishi Y, Nada S, et al. (2000) Transmembrane phosphoprotein $\mathrm{Cbp}$ regulates the activities of Src-family tyrosine kinases. Nature 404: 999-1003.

44. Torgersen KM, Vang T, Abrahamsen H, Yaqub S, Horejsí V, et al. (2001) Release from tonic inhibition of $\mathrm{T}$ cell activation through transient displacement of C-terminal Src kinase (Csk) from lipid rafts. J Biol Chem 276: 29313-29318.

45. Balagopalan L, Coussens NP, Sherman E, Samelson LE, Sommers CL (2010) The LAT story: a tale of cooperativity, coordination, and choreography. Cold Spring Harb Perspect Biol 2.

46. Torgersen KM, Vang T, Abrahamsen H, Yaqub S, Taskén K (2002) Molecular mechanisms for protein kinase A-mediated modulation of immune function. Cell Signal 14: 1-9

47. Pathak P, London E (2011) Measurement of lipid nanodomain (raft) formation and size in sphingomyelin/POPC/cholesterol vesicles shows TX-100 and transmembrane helices increase domain size by coalescing preexisting nanodomains but do not induce domain formation. Biophys J 101: 2417-2425.

Submit your next manuscript and get advantages of OMICS Group submissions

Unique features:

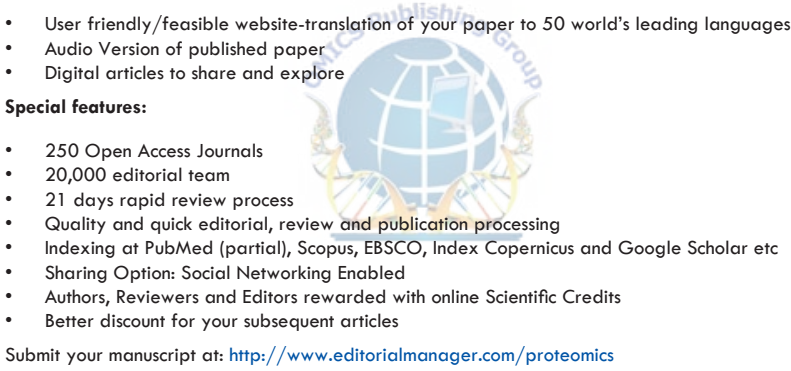

